\title{
Update on eating disorders: current perspectives on avoidant/restrictive food intake disorder in children and youth
}

This article was published in the following Dove Press journal:

Neuropsychiatric Disease and Treatment

19 January 2016

Number of times this article has been viewed

\author{
Mark L Norris' \\ Wendy J Spettigue ${ }^{2}$ \\ Debra K Katzman ${ }^{3}$ \\ 'Division of Adolescent Medicine, \\ Department of Pediatrics, Children's \\ Hospital of Eastern Ontario, \\ University of Ottawa, Ottawa, ON, \\ Canada; ${ }^{2}$ Department of Psychiatry, \\ Children's Hospital of Eastern \\ Ontario, University of Ottawa, \\ Ottawa, ON, Canada; ${ }^{3}$ Division of \\ Adolescent Medicine, Department of \\ Pediatrics, Hospital for Sick Children, \\ University of Toronto, Toronto, ON, \\ Canada
}

Correspondence: Mark L Norris Division of Adolescent Medicine, Department of Pediatrics, Children's Hospital of Eastern Ontario, University of Ottawa, 40I Smyth Road, Ottawa,

ON KIH 8LI, Canada

Tel + I 6137377600

Fax +l 6137384878

Emailmnorris@cheo.on.ca

\begin{abstract}
Avoidant/restrictive food intake disorder (ARFID) is a new eating disorder diagnosis that was introduced in the Diagnostic and Statistical Manual of Mental Disorders (DSM) fifth edition. The fourth edition of the DSM had failed to adequately capture a cohort of children, adolescents, and adults who are unable to meet appropriate nutritional and/or energy needs, for reasons other than drive for thinness, leading to significant medical and/or psychological sequelae. With the introduction of ARFID, researchers are now starting to better understand the presentation, clinical characteristics, and complexities of this disorder. This article outlines the diagnostic criteria for ARFID with specific focus on children and youth. A case example of a patient with ARFID, factors that differentiate ARFID from picky eating, and the estimated prevalence in pediatric populations are discussed, as well as clinical and treatment challenges that impact health care providers providing treatment for patients.
\end{abstract}

Keywords: avoidant/restrictive food intake disorder, ARFID, eating disorder, picky eating, prevalence, treatment

\section{Introduction}

Avoidant/restrictive food intake disorder, or ARFID, was introduced in the Feeding and Eating Disorders (EDs) section of the Diagnostic and Statistical Manual of Mental Disorders (DSM) fifth edition (DSM-5). ${ }^{1}$ The body of evidence on the characteristics, course, and outcome of children with "feeding disorders of infancy or early childhood" as defined in the fourth edition of the DSM (DSM-IV) is limited. This DSM-IV diagnosis relied on the presence of weight loss or failure to gain weight, and failed to account for circumstances that might allow a patient to stay adequately nourished as a result of the use of enteral feedings or oral nutritional supplements. ${ }^{2}$ Further, this diagnostic category was restricted to children less than 6 years, and put a substantial emphasis on negative or maladaptive interactions between the child and caregiver. In the years leading up to the DSM-5, it became apparent that there was a group of children, adolescents, and young adults who displayed feeding issues that did not fit into the diagnostic categories of anorexia nervosa $(\mathrm{AN})$ or bulimia nervosa $(\mathrm{BN})$. These patients were often given varying diagnoses including the residual diagnosis of ED not otherwise specified. Further, this patient population often required the expertise of a multidisciplinary treatment team to provide nutritional rehabilitation, medical management, and psychological treatment. The DSM-5 Eating Disorder Working Group recognized that this subset of individuals included children, adolescents, and adults and presented with histories of weight loss in the context of substantial restriction and often pronounced physiological and/or psychosocial distress. These patients were distinct from those with AN as they lacked 
body image preoccupation, fear of weight gain, or drive for thinness. Field studies were conducted to better describe this group. As such, the Working Group rearticulated the diagnosis of "EDs of infancy and early childhood" and named this new ED ARFID. At present, the body of literature that examines rates and presentation of ARFID in adult patients is extremely limited. As such, this article focuses on identification and management of pediatric patients.

\section{What is ARFID?}

ARFID was introduced in an attempt to capture a cohort of patients who struggle with impaired and distressing eating behaviors and symptoms and who lack weight and body image-related concerns associated with $\mathrm{AN}$ and BN. The diagnostic criteria of ARFID are outlined in the DSM-5. ${ }^{1}$ In summary, ARFID occurs in cases where patients exhibit restrictive or avoidant eating behaviors that result in significant weight loss, growth compromise, a reliance on nutritional supplements to meet daily energy requirements, nutritional deficiency (like iron deficiency anemia) or marked interference with the patient's psychosocial functioning. Patients with ARFID do not fear weight gain, are not dissatisfied with their body weight, shape, or size and lack any cognitions typically associated with anorexia nervosa. ARFID cannot be diagnosed in cases where the presence of a concurrent medical or mental health disorder can account for the behavior observed, but may be diagnosed if the severity of the eating disturbance exceeds that typically associated with the medical or psychiatric condition in question. ${ }^{1}$

Research that investigates the clinical utility and applicability of these diagnostic criteria is ongoing and will likely further inform future revisions of the DSM.

\section{Illustrative case example}

Susan (the patient's name has been changed to protect confidentiality) is a 10-year-old girl described by parents as always being an anxious child. Her past medical history was notable for a history of frequent stomach pains (without medical cause) and school refusal. Six months before being admitted to hospital, the patient developed recurrent viral gastroenteritis separated by 1 week's duration. The patient believed that the recurrence of symptoms was triggered by the resumption of eating, and complained of increased nausea, vomiting, and abdominal cramps whenever she ate. As a result, over the next few months she progressively ate less and lost weight. She was assessed and tested for a variety of medical illnesses (ie, food allergy, celiac disease, thyroid dysfunction, etc), but no pathology was identified. She lacked body image preoccupation, fear of weight gain, or drive for thinness. Her parents began to progressively eliminate foods that could potentially exacerbate her symptoms (ie, foods with gluten, dairy products) but with limited effect. She was eventually admitted to a local tertiary-care hospital where she underwent a gastroenterology assessment, including endoscopy, abdominal ultrasound, extensive blood work, and a dietitian consult. All medical testing was unremarkable and she was subsequently discharged. The patient continued to lose weight and was readmitted weeks later having lost $33 \%$ of her pre-morbid body weight.

She was hospitalized under the medical team but failed to gain weight. The hospital's multidisciplinary ED team was consulted and diagnosed Susan with ARFID. The diagnosis was made based upon the fact that the patient had demonstrated persistent failure to meet appropriate nutritional and/ or energy needs and had lost a significant amount of weight in the preceding months. The illness was causing significant impairment in multiple aspects of her life and could not be explained by culturally sanctioned practices, the presence of body image or weight concerns, or a concomitant medical condition. The patient was started on a treatment plan that consisted of regular family therapy, individual therapy targeting her anxiety, and olanzapine at bedtime; once weight improved, her anxiety was also treated with a selective serotonin reuptake inhibitor (fluoxetine).

The family therapist worked to raise parents' anxiety about the seriousness of the illness, and used this to mobilize parents to take control of Susan's nutritional intake. Early in treatment, the patient was noted to have regular temper tantrums, and to sob frequently during meals, complaining of severe abdominal pain. Susan's parents were empowered to stay firm and compassionate and help their daughter to eat what was expected. Slowly, the patient began to increase the amount of food eaten, which led to weight gain and eventually fewer temper tantrums. Parents were able to consistently increase food intake whenever weight gain slowed, targeting at least $1 \mathrm{~kg}$ of weight gain per week. Parents were empowered to spend as much time as possible out of hospital on passes with Susan and to help her take nutrition at home. Two months after starting family therapy, the patient was discharged and at this point in treatment was consuming almost 3,000 calories per day. One month later she reached her expected weight, at which point her nutrition was slowly tapered to prevent further weight gain. She was far less anxious, less labile, and no longer having temper tantrums. Her only medication was fluoxetine for anxiety. She gained insight and was able to identify that anxiety made her stomach hurt. Through individual therapy, she also learned 
some relaxation techniques. Parents were empowered to set goals of normalizing eating, including helping Susan to eat a variety of foods and to eat at restaurants. By the end of therapy Susan was normal weight (having gained $11 \mathrm{~kg}$ ), back to eating an appropriate amount of nutrition for her age, and was much calmer and more mature with better coping skills. She continued to be home schooled and participated in community-based sports.

\section{More than picky eating}

As ED experts sought to better understand the clinical characteristics associated with patients with ARFID, early media reports stated that the DSM-5 had moved to pathologize picky eating as a psychiatric condition. ${ }^{3}$ However, the ARFID diagnosis was meant to identify only those patients with clinically significant restrictive eating problems that resulted in persistent failure to meet an individual's nutritional and/or energy needs, thus eliminating many patients who are labeled as picky or fussy eaters. Part of the challenge is that there is no standardized definition for "picky eating". Picky eating is generally defined as occurring in children who are normal weight but consume an inadequate variety of foods through rejection of foods that may either be familiar or unfamiliar to them. ${ }^{4}$ Common characteristics include limitations in the variety of foods eaten, unwillingness to try new foods (food neophobia), and aberrant eating behaviors. ${ }^{4}$ Picky or fussy eating may include rejection of foods of a particular texture, consistency, color, or smell. Such food "neophobia" generally peaks between the 2 nd and 6 th year of life, with gradual reduction over time such that few are affected beyond their early adult years..$^{5-7}$ One of the challenges regarding studies on picky eating relates to the manner by which patients are identified, which in turn affects the degree of compromise and impairment reported from food-related behaviors. Studies have at times reported conflicting results depending on the specific population being studied. This has resulted in a very heterogeneous cohort that on one side of the spectrum has eating behaviors that are within the expected developmental trajectory for many normal children, and on the other side includes children who exhibit extreme behaviors and severe impairment, more in keeping with what would now be described as ARFID. Given these and other challenges related to epidemiological research, studies of picky eating have reported wide incidence and prevalence ranges, depending on the specific methodology employed. Prevalence rates for picky eating ranges from $14 \%$ to $50 \%$ in preschool children and $7 \%-27 \%$ in older children. ${ }^{8-13}$ Cardona Cano et al's recent population study on picky eating in children utilized two questions on the Children's Behaviour Checklist to establish a diagnosis of picky eating. ${ }^{13}$ It was assumed that this would capture all patients with picky eating, ranging from those who have a developmentally normative course to those left with significant impairment (and therefore possibly ARFID). At the age of 14 months, infants identified as being picky eaters ate less, had less variability in the amount of foods consumed, and had lower caloric intake than non-picky eaters. ${ }^{13}$ By the age of 4 years, picky eaters were rated as more fussy, with higher satiety responses, greater desires to drink fluids, less pleasure associated with eating, and overall lower food responsiveness compared to the matched controls. ${ }^{13}$ Of all the children sampled, $54.5 \%$ were classified as never picky eaters, $32.3 \%$ remitting picky eaters, $4.0 \%$ late-onset picky eaters, and $4.2 \%$ persistent picky eaters. ${ }^{13}$ Risk factors noted among the persistent picky eater group included male sex, low birth weight, non-Western maternal ethnicity, and lower parental income. ${ }^{13}$ It will be interesting to compare these results to epidemiological studies of children with ARFID. However, in the future it will be important that researchers undertaking nutritional and feeding studies in infants and children use standardized methodologies and definitions to ensure that results have applicability and can be compared ideally across populations.

\section{How common is ARFID?}

At present, few population studies in EDs have focused or reported on rates of ARFID; this is not surprising given that the DSM-5 was released in 2013. As with all epidemiological studies of EDs, there will be a number of challenges inherent to answering this question effectively, including challenges related to the types of studies and populations being studied (eg, population-based studies, case registries, profiles of patients attending clinics), the processes that are undertaken to make diagnoses (eg, clinical interviews, survey questions), and who develops the research questions (eg, ED experts, psychiatrists, developmental pediatricians, dietitians). It will also be important to better understand how eating problems present in different age groups. There has been very little research on rates of EDs in very young children. All of these factors make it difficult to know just how prevalent ARFID is in children and adolescents.

A British national surveillance study (2005-2006) documented the incidence of early-onset EDs using modified DSM-IV criteria as 3.01 cases per 100,000 of which $19 \%$ (0.57 cases per 100,000) of those diagnosed lacked body image issues or fear of weight gain. ${ }^{14}$ A Canadian national surveillance study (2003-2005) suggested that the incidence of early-onset EDs in 5- to 12-year olds was 
2.6 cases per 100,000 person-years. ${ }^{15}$ In this sample, $26.7 \%$ of cases diagnosed with EDs failed to endorse fears of getting fat or gaining weight, suggesting the possibility of an ARFID diagnosis $\left(0.69\right.$ cases per 100,000). ${ }^{15}$ Although a lower overall incidence of EDs was observed in those aged 5-9 years as compared to those aged 10-12 years, rates of age-specific ED behaviors were not provided. To date, there is only one community-based study of ARFID, which documented a point prevalence of $3.2 \%$ in a Swiss schoolbased sample of 1,444 children aged $8-13$ years using a self-report measure. ${ }^{16}$

The rates of ARFID have ranged from 5\% to $14 \%$ among pediatric inpatient ED programs and as high as $22.5 \%$ in a pediatric ED day treatment program. ${ }^{17-21}$ Studies have consistently demonstrated that, compared to those with AN or $\mathrm{BN}$, ARFID patients are younger, have higher proportion of males, and are commonly diagnosed with comorbid psychiatric and/or medical symptoms. ${ }^{17-20}$

Two non-ED clinical studies have also reported on rates and characteristics of ARFID patients. In the first, authors described clinical findings drawn from a case series of 29 patients presenting with pediatric acute-onset neuropsychiatric syndrome and discussed how features overlapped those outlined for ARFID. These patients showed some similarities to those drawn from ED samples in that affected children were young, had a high proportion of male patients (in fact, male to female ratio was 2:1), and also exhibited comorbid psychiatric symptoms. ${ }^{22}$ In the second study, researchers conducted a retrospective chart review of 2,231 consecutive new referrals to gastrointestinal specialty clinics in an attempt to understand how commonly patients with ARFID presented. They identified ARFID in 1.5\% of all patients assessed, but noted that some features of the diagnosis were present in an additional $2.4 \%$, suggesting that the criteria do not lead to over-inclusion of cases..$^{23}$ In this setting, patients were again more likely to be male $(67 \%))^{23}$ Although each of these studies adds a different piece to the puzzle, in combination they only offer us a very crude guess as to the prevalence rate of ARFID; well-designed prospective surveillance and population studies are required to provide a better understanding of the whole picture. The epidemiology of ARFID in the general non-clinical population remains unknown.

\section{Clinical and treatment challenges}

Patients with ARFID present with complicated and varied histories and risk factors that include varied medical and psychiatric factors affecting nutritional intake but with no body image concerns, making referrals to the most appropriate health care professional or facility challenging. Patients may be fearful and stressed, reacting to stress or trauma; reacting to messages about "dangerous" foods or chemicals (such as fat, sugar, or chemical additives); restricting to avoid pain, nausea, or risk of choking or vomiting; restricting to avoid adverse tastes or textures; or reacting to stressful emotions at meal times. This results in a variety of case presentations. Few hospitals have dedicated feeding programs that span the entire pediatric age group and so patients are often referred to a myriad of clinics depending on the age and presenting features. ${ }^{18}$ According to the authors' experience, many patients' first point of contact is usually with a family physician or general pediatrician. Other children may be referred to an occupational therapist, dietitian, developmental pediatrician, gastroenterologist, psychologist, psychiatrist, or adolescent health physician. The unpredictable referral and treatment patterns for these cases increase the likelihood that patients will be left with a vague diagnosis and disjointed care plan that lacks the kind of specialized coordinated care that is required to optimize successful outcomes. Clearly, given the potential heterogeneity of the clinical presentation of this population, it is critical for health care providers to have an understanding of the varied presentations of children and adolescents with ARFID, so they can best diagnose and develop appropriate treatment recommendations. At present there are no evidence-based treatment recommendations for ARFID; however, clinical experience suggests that patients' needs might differ depending on what factors are thought to be driving the distress and eating disturbances. As an example, patients who present with pronounced food restriction and weight loss that has occurred as a result of a fear of choking may respond best to cognitive strategies to help address these underlying fears. On the other hand, young children who present with longstanding histories of poor growth as a consequence of severe selectivity may utilize strategies that involve a combination of psychological and behavioral approaches.

Given the lack of empirical data on the treatment strategies of ARFID, best practice treatment guidelines have not yet been developed, which potentially increases the risk of prolonged resource-intensive hospital stays for complex cases. Interestingly, a recent review examined multisite ED outcome trajectories and demonstrated that patients with ARFID were less likely to be followed for 1-year duration, despite the fact that ARFID patients fared no better with weight recovery than the other ED groups. The authors suggested that one possible reason for this difference may be 
related to the fact that patients with ARFID were referred into different therapy modalities outside that offered by the ED team. ${ }^{21}$ Further, because the study population was younger, it is also possible that patients were followed by providers outside of traditional adolescent medicine clinics. Another recently published retrospective review revealed that ARFID patients were more likely than those with AN to be admitted at lower weights relative to estimated healthy weight, struggle more with weight gain in hospital, rely on enteral nutrition during inpatient hospitalizations, have longer hospital stays, and require rates of readmission within 1 year that mirrored those with AN. ${ }^{24}$ Further, patients with ARFID recovered at a rate similar to patients with $\mathrm{AN}$, although $38 \%$ of the sample continued to struggle in some meaningful way 1 year after initial diagnosis. ${ }^{24}$

\section{Future directions}

Now that ARFID has been identified and defined, researchers need to focus on determining prevalence rates, outlining risk factors, describing patient demographics and case presentations, comparing different treatments, studying the effectiveness of medications, and describing the course of illness and factors that affect outcomes in this patient population. Studies are required that better define how this illness presents across the entire life span. Given the reality that many patients with ARFID have complex presentations that often require specialized treatment, it will be important that clinicians be educated about ARFID, have knowledge of the diagnostic characteristics of the illness, and have an understanding of how a patient's needs should be managed. Currently, there are no prospective studies that have reported outcomes on interventions that have targeted patients with ARFID. As these evidence-based treatments become available, it will be important to apply treatments that optimize outcomes in hopes of minimizing morbidity associated with the illness.

\section{Disclosure}

The authors report no conflicts of interest in this work.

\section{References}

1. American Psychiatric Association. Diagnostic and Statistical Manual of Mental Disorders. 5th ed. Arlington, VA: American Psychiatric Publishing; 2013.

2. Bryant-Waugh R, Markham L, Kreipe RE, Walsh BT. Feeding and eating disorders in childhood. Int J Eat Disord. 2010;43(2):98-111.

3. Nationalpost.com. Picky eaters could join ranks of mentally ill. Available from: http://news.nationalpost.com/news/canada/picky-eaters-couldjoin-ranks-of-mentally-ill. Accessed September 15, 2015.
4. Dovey TM, Staples PA, Gibson EL, Halford JC. Food neophobia and 'picky/fussy' eating in children: a review. Appetite. 2008;50: 181-193.

5. Addessi E, Galloway AT, Visalberghi E, Birch LL. Specific social influences on the acceptance of novel foods in 2-5-yearold children. Appetite. 2005;45:264-271.

6. Cashdan E. A sensitive period for learning about food. Human Nature. 1994;5:279-291

7. McFarlane T, Pliner P. Increased willingness to taste novel foods: effects of nutrition and taste information. Appetite. 1997;28:227-238.

8. Marchi M, Cohen P. Early childhood eating behaviors and adolescent eating disorders. J Am Acad Child Adolesc Psychiatry. 1990;29: $112-117$.

9. Mascola AJ, Bryson SW, Agras WS. Picky eating during childhood: a longitudinal study to age 11 years. Eat Behav. 2010;11:253-257.

10. Dubois L, Farmer A, Girard M, Peterson K, Tatone-Tokuda F. Problem eating behaviors related to social factors and body weight in preschool children: a longitudinal study. Int J Behav Nutr Phys Act. 2007;4:9.

11. Carruth BR, Ziegler PJ, Gordon A, Barr SI. Prevalence of picky eaters among infants and toddlers and their caregivers' decisions about offering a new food. $J$ Am Diet Assoc. 2004;104:s57-s64.

12. Micali N, Simonoff E, Elberling H, Rask CU, Olsen EM, Skovgaard AM. Eating patterns in a population-based sample of children aged 5 to 7 years: association with psychopathology and parentally perceived impairment. J Dev Behav Pediatr. 2011;32:572-580.

13. Cardona Cano S, Tiemeier H, Van Hoeken D, et al. Trajectories of picky eating during childhood: a general population study. Int J Eat Disord. 2015;48(6):570-579.

14. Nicholls DE, Lynn R, Viner RM. Childhood eating disorders: British national surveillance study. Br J Psychiatry. 2011;198(4):295-301.

15. Pinhas L, Morris A, Crosby RD, et al. Incidence and age-specific presentation of restrictive eating disorders in children: a Canadian Paediatric Surveillance Program study. Arch Pediatr Adolesc Med. 2011;165(10): 895-899.

16. Kurz S, van Dyck Z, Dremmel D, Munsch S, Hilbert A. Earlyonset restrictive eating disturbances in primary school boys and girls. Eur Child Adolesc Psychiatry. 2015;24(7):779-785.

17. Norris ML, Robinson A, Obeid N, Harrison M, Spettigue W, Henderson K. Exploring avoidant/restrictive food intake disorder in eating disordered patients: a descriptive study. Int J Eat Disord. 2014; 47(5):495-499.

18. Fisher MM, Rosen DS, Ornstein RM, et al. Characteristics of avoidant/ restrictive food intake disorder in children and adolescents: a "new disorder" in DSM-5. J Adolesc Health. 2014;55(1):49-52.

19. Ornstein RM, Rosen DS, Mammel KA, et al. Distribution of eating disorders in children and adolescents using the proposed DSM-5 criteria for feeding and eating disorders. $J$ Adolesc Health. 2013;53(2): 303-305.

20. Nicely TA, Lane-Loney S, Masciulli E, Hollenbeak CS, Ornstein RM. Prevalence and characteristics of avoidant/restrictive food intake disorder in a cohort of young patients in day treatment for eating disorders. Int J Eat Disord. 2014;2(1):21.

21. Forman SF, McKenzie N, Hehn R, et al. Predictors of outcome at 1 year in adolescents with DSM-5 restrictive eating disorders: report of the national eating disorders quality improvement collaborative. $J$ Adolesc Health. 2014;55(6):750-756.

22. Toufexis MD, Hommer R, Gerardi DM, et al. Disordered eating and food restrictions in children with PANDAS/PANS. $J$ Child Adolesc Psychopharmacol. 2015;25(1):48-56. doi:10.1089/cap.2014.0063.

23. Eddy KT, Thomas JJ, Hastings E, et al. Prevalence of DSM-5 avoidant/ restrictive food intake disorder in a pediatric gastroenterology healthcare network. Int J Eat Disord. 2015;48(5):464 470. doi:10.1002/eat.22350.

24. Strandjord SE, Sieke EH, Richmond M, Rome ES. Avoidant/ Restrictive Food Intake Disorder: Illness and Hospital Course in Patients Hospitalized for Nutritional Insufficiency. J Adolesc Health. 2015;57(6):673-678. 


\section{Publish your work in this journal}

Neuropsychiatric Disease and Treatment is an international, peerreviewed journal of clinical therapeutics and pharmacology focusing on concise rapid reporting of clinical or pre-clinical studies on a range of neuropsychiatric and neurological disorders. This journal is indexed on PubMed Central, the 'PsycINFO' database and CAS,

and is the official journal of The International Neuropsychiatric Association (INA). The manuscript management system is completely online and includes a very quick and fair peer-review system, which is all easy to use. Visit http://www.dovepress.com/testimonials.php to read real quotes from published authors.

Submit your manuscript here: http://www.dovepress.com/neuropsychiatric-disease-and-treatment-journal 